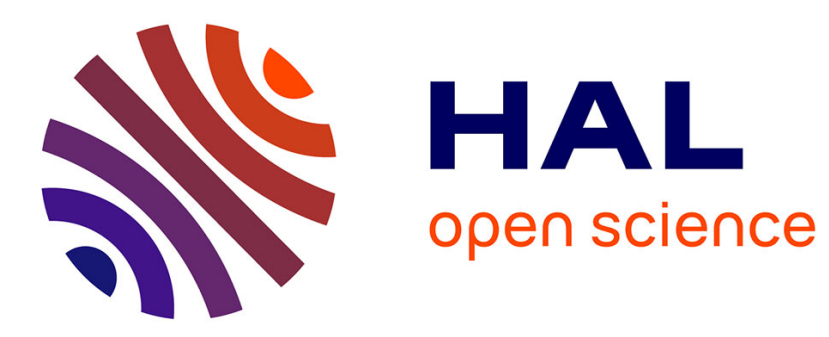

\title{
Survey on the state of systems and control
}

Vincent Blondel, M. Gevers, A. Lindquist

\section{To cite this version:}

Vincent Blondel, M. Gevers, A. Lindquist. Survey on the state of systems and control. [Research Report] RR-2479, INRIA. 1995. inria-00074195

\section{HAL Id: inria-00074195 https://hal.inria.fr/inria-00074195}

Submitted on 24 May 2006

HAL is a multi-disciplinary open access archive for the deposit and dissemination of scientific research documents, whether they are published or not. The documents may come from teaching and research institutions in France or abroad, or from public or private research centers.
L'archive ouverte pluridisciplinaire HAL, est destinée au dépôt et à la diffusion de documents scientifiques de niveau recherche, publiés ou non, émanant des établissements d'enseignement et de recherche français ou étrangers, des laboratoires publics ou privés. 


\title{
I N R I A
}

INSTITUT NATIONAL DE RECHERCHE EN INFORMATIQUE ET EN AUTOMATIQUE

\section{Survey on the state of systems and control}

\author{
V. BLONDEL - M. GEVERS - A. LINDQUIST
}

$$
\mathrm{N}^{\circ} 2479
$$

Janvier 1995

PROGRAMME 5

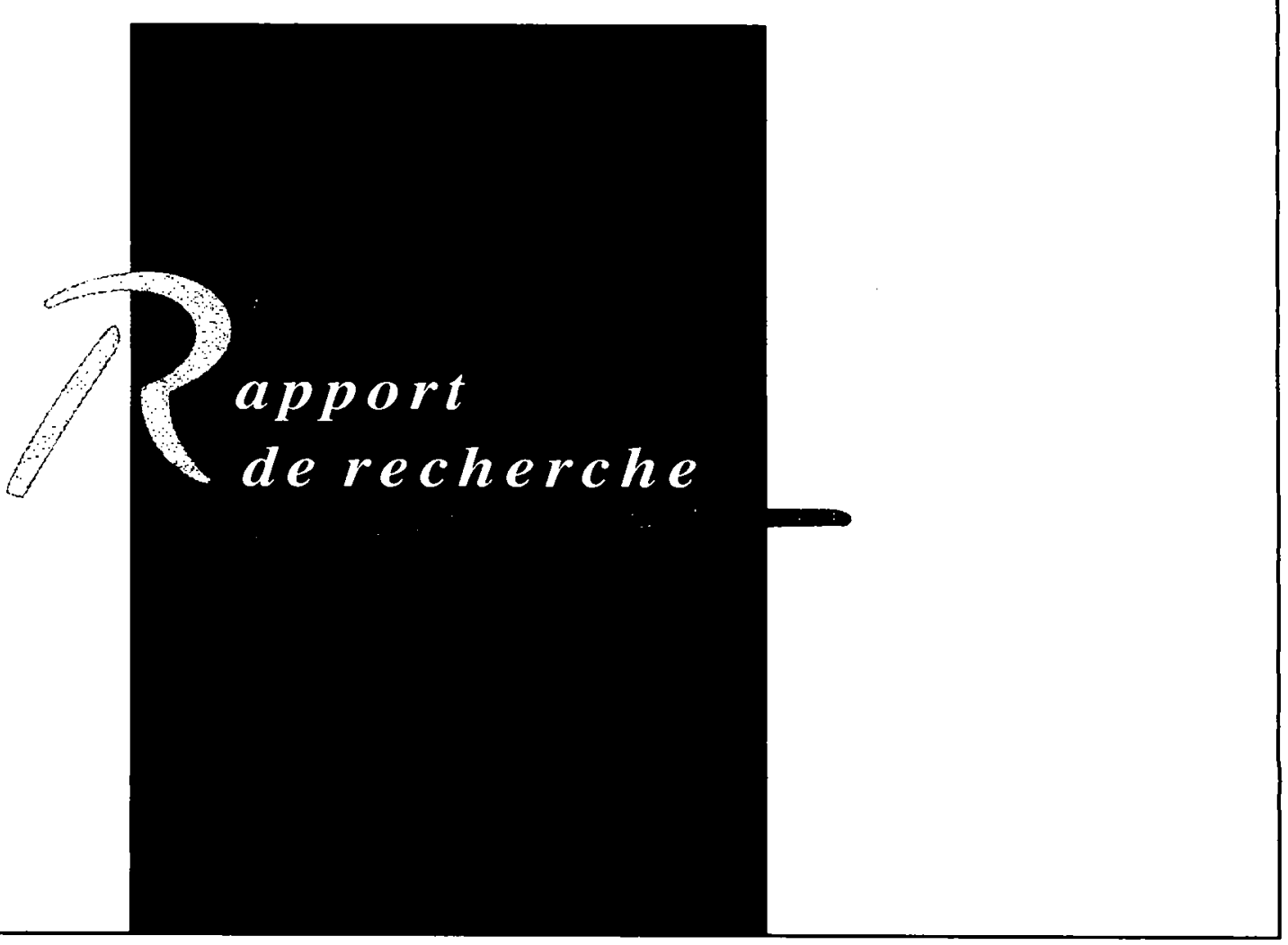


Les rapports de recherche de l'INRIA INRIA research reports sont disponibles en format postscript sous are available in postscript format ftp.inria.fr (192.93.2.54) ftp.inria.fr (192.93.2.54)

si vous n'avez pas d'accès ftp if you haven't access by ftp la forme papier peut être commandée par mail : we recommend ordering them by e-mail : e-mail :dif.gesdif@inria.fr e-mail :dif.gesdif@inria.fr (n'oubliez pas de mentionner votre adresse postale). (don't forget to mention your postal address).

par courrier : by mail :

Centre de Diffusion Centre de Diffusion

INRIA INRIA

BP 105 - 78153 Le Chesnay Cedex (FRANCE) BP 105 - 78153 Le Chesnay Cedex (FRANCE) 


\title{
Survey on the state of systems and control
}

\section{Enquète sur l'état de la théorie des systèmes et du contrôle}

\author{
V. Blondel \\ INRIA Rocquencourt, Domaine de Voluceau BP105, F-78153 Le Chesnay Cedex, France \\ M. Gevers \\ Université Catholique de Louvain, Cesame, Bâtiment Euler, B-1348 Louvain-la-Neuve, Belgium
}

\section{A. Lindquist}

Royal Institute of Technology, Department of Mathematics, Division of Optimization and Systems Theory, S-100 44 Stockholm, Sweden

Abstract. This report contains the answers of twenty-seven experts in system and control to the three questions:

1. On what problems in control systems theory have you been working during the last five years?

2. What do you see as the major open problems in control systems theory?

3. What do you think are the major challenges that face the control engineering community in the next few years?

Résumé: Ce rapport est un recueil des réponses données par vingt sept experts en théorie des systèmes et du contrôle aux trois questions suivantes:

1. A quels problèmes de la théorie des systèmes et du contrôle avez vous travaillé durant ces cinq dernières années?

2. Quels sont à votre avis les problèmes ouverts les plus importants en théorie des systèmes et du contrôle?

3. Quels sont à votre avis les défis majeurs auxquel devra faire face la communauté du contrôle dans les prochaines années? 


\title{
Survey on the state of systems and control*
}

\author{
Vincent Blondel $^{\dagger} \quad$ Michel Gevers $^{\ddagger} \quad$ Anders Lindquist $^{\S}$ \\ January 31,1995
}

Contributors: Ackermann J., Anderson B. D. O., Araki M., Åström K. J., Balchen J., Barmish B. R., Bensoussan A., Byrnes C. I., Caines P. E., Chen H. F., Davis M. H. A., Davison E. J., Fliess M., Goodwin G. C., Kimura H., Kučera V., Laub A. J., Ljung L., McFarlane A. J. G., Picci G., Pozniak A. S., Skelton R. E., Tsypkin Y. Z., Varaiya P. P., Vidyasagar M., Willems J. C., Wonham W. M.

\section{Introduction}

During 1994 forty-one experts in control systems theory were asked to give brief answers to the following three questions:

1. On what problems in control systems theory have you been working during the last five years?

2. What do you see as the major open problems in control systems theory?

3. What do you think are the major challenges that face the control engineering community in the next few years?

Here we publish the twenty-seven answers that we received (a $66 \%$ success rate). Except for spelling and typographical modifications, answers are published unedited. In the sections that precedes the list of contributions received, we have attempted to describe the major trends that emerge from the answers to the second and third questions.

In compiling the list of people contacted for the questionnaire, we have tried to keep a balance between the major regions where control research is active and between the major fields of research in systems and control. The list does certainly not include all key actors in systems and control; its sole ambition is to be reasonably representative of the various trends present in the community.

Those readers interested in other descriptions of challenges and open problems in systems and control may want to consult the three references given after our conclusion.

\footnotetext{
*The production of this paper has been partly funded by the Belgian Programme on Interuniversity Poles of Attraction, initiated by the Belgian State, Prime Minister's Office for Science, Technology and Culture. The scientific responsibility rests with its authors.

${ }^{\dagger}$ INRIA Rocquencourt, Domaine de Voluceau BP 105, F-78153 Le Chesnay Cedex, France

${ }^{\ddagger}$ Université Catholique de Louvain, Cesame, Bâtiment Euler, B-1348 Louvain-la-Neuve, Belgium

${ }^{\S}$ Royal Institute of Technology, Department of Mathematics, Division of Optimization and Systems Theory, S-100 44 Stockholm, Sweden
} 


\section{Major open problems}

Most of the respondents have proposed lines of research rather than specific open problems. We have identified five themes in the answers.

\section{Specific problems: output feedback stabilization and other problems}

The most often mentioned specific open problem (proposed by Davison, Kimura and Kucera) is the output feedback stabilization problem. Kučera's question is: "[What are] the limits of static output feedback in altering the dynamics of linear systems?". Kimura asks for a solution of the problem that does not make use of "heavy mathematics like algebraic geometry" whereas Davison's concern is to obtain a "tractable algorithm". Let us mention here that these three contributors have significantly contributed to this problem in the 70's. Other proposed major open problems include the problem of obtaining "the minimal order of a stabilizing compensator for a given plant" (Kimura), the development of a "Kharitonov theory for the matrix case" (Davison), efficient numerical methods for $H_{\infty}$ control design (Chen, Kimura), and a robust control design problem: "Given a plant family defined by a model structure and parameter ranges, find a necessary and sufficient condition for the existence of a fixed-gain or simply gain-scheduled controller that yields closed-loop stability" (Ackermann).

\section{Adaptive vs. robust control: there is a need for a grand synthesis}

For Davis the major open problem is clearly identified: "Surely, the grand challenge is to make adaptive control work". Adaptive control is also one of the questions raised by Kimura who asks for "a simple adaptive control scheme that works for most plants in practice". Araki's wish is to achieve "deeper understanding, a simpler mechanism [and] a more powerful mechanism [for adaptive control]". More specific are the problems of Pozniak ("Construct information inequalities for adaptive control problems") and Kimura ("Establish the intrinsic necessity of adaptive controllers based on the clear demonstration of the performance limit of linear timeinvariant controllers"),

Problems related to the link between adaptive and robust techniques appear in several contributions. Anderson's and Araki's questions are almost identical: "When to use robust (and how to use it) and when to use adaptive control, and when to use a mixture [...] ?" (Anderson). In the same vein Pozniak asks for "robust-adaptive and adaptive-robust control theories" and Chen asks for a theory of "adaptive control for partially observed systems". According to Davis: "There are asymptotic theories that are completely non-robust, theories of robust control that are non-adaptive, and various other pieces of the jigsaw puzzle, but no grand synthesis". A similar comment is given by Caines "There is not as yet an adequate robust adaptive control theory". Caines also ventures an explanation for this state-of-affairs: "this may be due to the fact that there is a complete mismatch between the current mathematical formulations of robust and adaptive control".

Robust control issues are raised, among others, by Tsypkin, Chen and Picci. As Kučera puts it: "Future control theories will have to handle families of models to account for mismodelling and produce reliable control systems". A main concern in this respect is that of finding an appropriate way to model uncertainty. Willems asks for "[a new paradigm for] modelling uncertainty, abandoning the unrealistic stochastic approach, and less simplistic than small gains or hard parameter bounds". For Skelton "a fundamental limitation of robust control theory is the presumption that the model errors are independent of the input. Available control techniques do not come to grips with the fact that the modeling problem and the control problem are not independent". In the same direction, Kimura sees "a design philosophy in which the quality of our knowledge is explicitly taken into account" as one of the main challenges for the control community. Similar concerns are also present in Vidyasagar's and Picci's answers. 


\section{Nonlinear systems: almost everything, but things that work!}

One of Davison's major open problems goes right to the point: "Nonlinear system theory: almost everything [is open]". This is in line with Davison's other comment: "I believe control theory is just in its infancy". Nonlinear control is mentioned by several other contributors as being one of the main open problems in control (e.g., see Tsypkin, Barmish, Willems, Araki). A recurring concern of those mentioning nonlinear systems is that of finding practical methods. For Åström "We need a much better understanding of nonlinear systems and we need design methods for them". The same spirit is present in Chen's contribution in which he asks for "practically applicable methods for controlling nonlinear systems" and in Araki's suggestion: "We could find specific (probably narrower but more nonlinear than those studied at present) classes of nonlinear systems which are essentially important in applications, and develop an individual theory for each class". For Byrnes one should search for "a systematic methodology for taking advantage of genuinely nonlinear effects to shape, or influence, the response of complex dynamical systems". Connected to the behavioral approach is also Willems's desire to see the emergence of a "nonlinear system theory that does not start from an input-output setting". A comment on the behavioral approach is also present in Fliess's answer.

\section{Hybrid systems: find a way to describe the interactions between the continuous and discrete worlds}

As Caines puts it: "Contemporary systems and control does not have theories that deal with the interaction of continuous systems (often the controlled system) and discrete systems (often the controller)". The need for such a theory is clearly identified by various contributors. Willems's wish is to have a "theory of integrated design in which the design of the controller and the plant are part of the same picture". Similar but more general is the desire of Ljung "to come up with a model concept which incorporates the discrete elements (hybrid, logical, sequential) in our traditional model world". In Åström's judgement "The whole area of mixed discrete and continuous time systems represent a lot of challenges. I personally believe that totally new approaches are needed". If Aström's judgment is to be taken seriously then there is plenty of room here for a Kalman of the nineties! Related to these discrete-continuous issues is Varaiya's warning: "One urgent issue is how to formalize symbol-processing, embedded, controllers within a continuous process". According to Caines a unified formulation "will require a fusion of systems and control, logic, and computer science". This of course relates to the links between control and computer science, a theme which is described below as one of the major challenges for the future.

\section{Other major problem areas}

Among the other major open problem areas suggested by more than one contributor we mention problems involving large systems (Bensoussan, Wonham: "Most problems involving large systems are open"), identification problems (Chen, Kimura, Picci: "Identification needs a lot of research"), and discrete event dynamical systems (Anderson, Chen).

\section{Major challenges}

1. New applications, new blood, fresh research problems... or die

Novelty is needed in the control community; as diagnosed by Anderson, there is a need to "re-investigate a now relatively mature discipline, via one or both of: applications, or major theoretical shifts of attention, say to discrete event systems". Otherwise, as Barmish predicts: "Without the injection of new blood into the field, we are in grave danger".

The need to "go to a wider sphere of applications" (Davis) is identified in several contributions as one of the main challenges for the control community. Examples of suggested fields of ap- 
plications include smart materials, health, environment, transportation and energy problems (Ackermann), manufacturing engineering (Araki), environment (Åström), thermo-nuclear processes and health (Pozniak). Byrnes also suggests that we "incorporate biological systems into our world view at a similar level as we have incorporated electrical and mechanical systems". Many contributors feel that the control community "should be somewhat more adventurous and outward-looking" (Wonham). According to Araki "people in this community should be positive in exploring new fields". The same idea is phrased in a more provocative way by Davis: "get out of the ghetto"! For Davis "Control is not like Physics. It is like Statistics: a service industry that exists to provide solutions to problems in a wide range of application areas". For Davison one should "relate control system theory and engineering to other fields in a more efficient way, e.g., computer sciences, biological systems, management science, signal analysis, banking systems, intelligent system theory". Other examples of related fields are given by McFarlane: "relate more closely to cognate fields - information sciences, computational and cognate science, artificial intelligence, and physiology and neurophysiology". Ljung's advice is similar: "To have a broad and open mind about control and feedback: Not to focus on the traditional control loops and leave the rest to the computer scientists and software engineers".

To conclude this section let us hope with Tsypkin that with this new blood "there will appear new points of growth in control theory".

\section{The gap and the elusive bridge}

Several contributors think of bridging the notorious "gap between theory and practice" (Bensoussan) as one of the main challenges for the future. For Goodwin: "It is certainly true that strong theory has been, and must remain, the cornerstone of Control Engineering. However, our greatest challenge is to better communicate the relevance of this theory to practical design issues". Fliess comments: "Some of the existing theories do not seem to be really helpful in applications. A major challenge is to understand why and to propose remedies". Chen's observation is that: "Practical problems arising from engineering, as a rule, do not meet the conditions required in the theory, and a sophisticated theory is not easy to be accepted by practitioners". As a consequence there "is a desperate need to develop new appropriate relevant theory" (Davison). For Åström "We have to decide to what extent we will contribute to applications" but if we want to contribute, as Åström wishes, then we need to "transfer ideas into products" (Byrnes). For Davis, those partly responsible for this "unhealthy situation" are the "control journals particularly the most prestigious ones - [that] have far too many papers addressing internal questions that, it is obvious, will never be of even indirect interest or relevance to the world outside".

\section{Computers, computers,...}

Computers are present everywhere in the answers to the questionnaire. For Balchen one should "bring more of the 1970-1990 control theoretic achievements into practical use utilizing the tremendous increase in computer capacity and performance". Davis's comment is: "I get the impression - perhaps wrongly - that the control community has been rather unambitious in its utilization of modern computer resources". Similar are McFarlane's and Laub's major open problems (McFarlane: "To absorb the implications of parallel processing for applied control", Laub: "Developing the next generation of (parallel and vector) algorithms, computer codes, and user interfaces for computer assisted control and system design"). Connected to implementation issues is Vidyasagar's slightly provocative question: "Up to now, we have proceeded on the assumption that a linear time-invariant control law is easier to implement than any other. What happens if this fundamental assumption is challenged?".

Another major challenge for the control community is to "make efficient use of results from computer science" (Ackermann). An example is given by Wonham: "To gain a much better understanding of computational complexity - what is and what is not tractable and what can 
and cannot be done?". In the same vein Bensoussan suggests to "[incorporate] computer science methods without losing leadership". The relations between the control and the computer engineering communities are pictured by Balchen: "The control engineering community has had a problem with its interaction with the computer science community. These two branches are to an increasing extent working on overlapping problems. This has led to confusion, inefficiency and even conflict". All these comments confirm Wonham's prediction: "Clearly the control science and engineering community will have to move closer to computer science and engineering". We finally quote Vidyasagar to conclude this section: "The major challenge faced by the control engineering community at present is coping with the interface between control and computing".

\section{An integrated view of control design}

A major challenge that comes forward repeatedly in the responses is that of developing a "general picture" of control, a "coherence among the wide range of disparate approaches in current use" (McFarlane). For Åström: "Systematic approaches to control system design that take a balanced view of the whole design problem are badly needed". More specific is Ackermann's desire of "design feedback systems based on complex information (e.g., contour extraction online from TV images) and smart materials with distributed sensors and actuators". According to Skelton "we are near the limits of performance that can be obtained by isolated theories and the sequential steps of plant design, model development, sensor-actuator selection, control design, controller implementation. The control community must develop scientific procedures for integrating (and yes iterating) these disciplines in a scientific way". In line with this analysis is Varaiya's major open problem: "How to use some of the key concepts of control - information, feedback, algorithms - in the formulation of a control architecture for realistic, complex systems".

\section{Conclusion}

When researchers in our community are asked to pinpoint the major open problems in systems and control theory, they do not take this question like mathematicians would. Save for a few precisely formulated questions, mostly related to output feedback, the answers have been more in terms of major problem areas where there is a perceived need for intense research efforts. The suggested major problem areas cover a rather large spread, thus reflecting the personal bias of each individual respondent.

On the other hand, there is a much stronger consensus on the question of what our major challenges are. These can be summarized as follows: we need to be more adventurous in our research and venture into new areas, our theory must be more relevant and helpful in applications, we need to undertake a major pedagogical effort in unifying and simplifying our concepts and design tools, and we must do so by employing to the fullest the new tools and methods delivered by computer science. Surprisingly perhaps, these recipes for the future are motivated by two widely divergent views about the state of our field among our panelists. Some believe that systems and control theory has now reached maturity, and that the only alternative to venturing into new areas is for our community to shrink or at least to loose out on the best students in the next generation. Others, on the contrary, believe that our field is still in its infancy and that the major theoretical and methodological challenges are ahead of us. Who has the best predictor? Only the future will tell...

\section{References}

Challenges to Control: A Collective View, IEEE Trans. of Automat. Control, vol. 32, n. 4, pp. 275-285, 1987. 
Future Directions in Control Theory: A Mathematical Perspective, SIAM Report, Philadelphia, 1989.

Essays on Control: Perspectives in the Theory and its Applications, H. L. Trentelman and J. C. Willems (eds), Birkhäuser, 1993.

\section{Acknowledgements}

The three authors wish to express their sincere gratitude to all those who have spent some of their precious time to provide answers to their questions. The authors are also thankful to I. Landau for his support throughout the completion of this project. 
Ackermann J. (Institut für Robotik und Systemdynamik, DLR, Wessling, Germany)

Past research

Problems of robust control systems analysis and design, in particular nonconservative methods for robustness with respect to uncertain physical parameters whose influence is traced through modelling and controller structure assumption into the closed-loop characteristic polynomial. A model that I studied in detail is that of four-wheel car steering with nonlinear tire characteristics. A feedback structure using a gyro and an accelerometer was derived that assures robust decoupling of lateral and yaw motions, robust steering transfer function one within the bandwidth of driver commands and velocity-independent well-damped yaw motion.

\section{Major open problems}

Given a plant family defined by a model structure and parameter ranges, find a necessary and sufficient condition for the existence of a fixed-gain or simply gain-scheduled controller (of assumed structure) that yields closed-loop stability.

\section{Major challenges}

1. Design feedback systems based on complex information (e.g. contours extracted on-line from TV images) and smart materials with distributed sensors and actuators.

2. Make efficient use of results from computer science.

3. Modelling, analysis and design of feedback systems for new applications in health, environment, transportation and energy problems. 
Anderson B. D. O. (Department of System Engineering, Australian National University, Canberra, Australia)

\section{Past research}

Adaptive control, robust control, practical controller realisation.

Major open problems

Knowing when to use robust control (and how to use it) and when to use adaptive control, and when to use a mixture, for a plant containing uncertainty including parametric uncertainty.

\section{Major challenges}

Re-investigate a now relatively mature discipline, via one or both of: applications, or major theoretical shifts of attention, say to discrete event systems. 
Araki M. (Department of Electrical Engineering II, Kyoto University, Tokyo, Japan)

Past research

1. Multirate digital controllers. Basic analysis of systems including samplers and holds which operate with different periods. Applications of these controllers to pole assignment and simultaneous stabilization problems.

2. Frequency domain theory for sampled-data control systems. Proposal of a method to analyse (and to design in future) intersample responses of digital control systems in the frequency domain. The key idea is to use an operator from $l_{2}$ to $l_{2}$ to represent the frequencydomain characteristics of sampled-data controllers.

Major open problems

1. Digital control. To obtain transparent understanding of digital control problems. I mean, to understand the problem of "digitalization in time (sampling)" and of "digitalization in space (quantization)" deeply, and preferably in a unified or interrelated way.

2. Adaptive control. Deeper understanding, simpler mechanism, more powerful mechanism, etc.

3. Theory of manufacturing engineering. To develop a theory, comparable to the feedback control theory, which can deal with pratical problems in manufacturing engineering, design of manufacturing factories, etc.

\section{Major challenges}

1. The digital control problems, the adaptive control problems and manufacturing engineering problems mentioned in my answer to the second question.

2. Application to new fields where the feedback control technique has not been fully utilized.

3. People in this community should be positive in exploring new fields. For instance, we could find specific (probably narrower but more nonlinear than those studied at present) classes of nonlinear systems which are essentially important in applications (not necessarily in the field of control engineering but in any field), and develop an individual theory for each class. 


\section{Åström K. J. (Department of Automatic Control, Lund Institute of Technology, Lund, Sweden)}

Past research

Over the past five years I have been working on adaptive control, automatic tuning of simple controllers, PID control, nonlinear controller and computer aided control engineering. You may be surprised over PID control but the majority of control loops in industry are in fact controlled by such devices and current industrial practice is by no means perfect. The work is by no means revolutionary from the theoretical point of view, but it has been very well received by industry and it has found its way into a large number of devices. The work on computer aided control engineering has focused on models described by differential algebraic systems. The work on nonlinear systems is geared towards specific nonlinear problems that arise in connection with friction, backlash, fast set point response etc. Very little of this is yet published.

\section{Major open problems}

Systematic approaches to control system design that takes a balanced view of the whole design problem are badly needed. We should also consider problems related to implementation issues. We need a much better understanding of nonlinear systems and we need design methods for them. The whole area of mixed discrete and continuous time systems represent a lot of challenges. I personally believe that totally new approaches are needed. We need a system theory that can capture the key issues that are involved in batch control.

\section{Major challenges}

We have to consider our environment. We need to be more responsive to industrial needs, we need to attract the brightest of a shrinking group of students, we need better positioning in academia. How do we do this? A compactification of current knowledge is badly needed. We need better and fewer books and courses. We have to decide to what extent we will contribute to applications. Personally I think that we should pursue this very actively. If not, our domain and outlook will shrink and we will drive towards the position circuit theory was in a long time ago. We need good fresh research problems to attract the really good students. If we loose on this account we will end up in the doldrums. 
Balchen J. G. (Institutt for teknisk kybernetikk, University of Trondheim, Norway)

Past research

1. A method for determining the stability of mxm multivariable process control systems.

2. State space predictive control of multivariable dynamic processes utilizing control vector parametrization and standard packages for constrained optimization. The algorithm contains a real time state space model for state and parameter estimation and a fast time model with identical structure for control vector calculations. The method is very well suited for both process control and vehicle control where the systems are highly nonlinear and constrained.

3. Elementary nonlinear decoupling, which is a version of model based input-output linearization and decoupling avoiding the use of differentiation of the output variables. A method for resolving the problem of input saturation by elementary nonlinear decoupling has been developed.

\section{Major open problems}

I do not see "one major open problem" but rather a whole family of problems related to the analysis and design of complex model based control systems. Since control algorithms are model based to incorporate a priori knowledge about system structure, mechanisms and parameters, a new approach is needed for "computer aided modeling of dynamic processes based on first principles". One "control systems theory" problem in this respect is a systematic procedure to determine the "optimum" model complexity in order to achieve a defined task of the ultimate control system.

\section{Major challenges}

$c$

The control engineering community has had a problem with its interaction with the computer science community. These two branches of science and engineering have developed partly quite independently but are to an increasing extent working on overlapping problems. This has lead to confusion, inefficiency and even conflict. A challenge will be to integrate the parts of the two fields that are overlapping by cross fertilization at the university education level and in basic research.

Another challenge is to bring more of the 1970-1990 control theoric achievements into practical use utilizing the tremendous increase in computer capacity and performance. 
Barmish B. R. (Department of Electrical and Computer Engineering, University of Wisconsin, Madison, USA)

\section{Past research .}

The main focal point of my recent research has been robustness analysis with structured real parametric uncertainty. This research area was sparked by Kharitonov's Theorem and seems to have reached a plateau in the following sense: Robust stability theory with affine dependence on parameters has now reached a mature state. We are now entering a new phase involving more complicated nonlinear dependence on parameters. In order to address robustness problems involving nonlinear dependence on parameters, we now see a number of researchers emphasizing mathematical programming instead of analytical solution.

\section{Major open problems}

An important (and often unrecognized) component of research is asking the right question and formulating the problem in such a way that it is both mathematically tractable and important in an engineering sense. In other words, it may well be the case that the "major open problem in control" is yet to be formulated. To clarify my response, let's consider an example: The classical linear quadratic regulator is important because both the question and the answer appear in the same paper. More recently, a second example is provided via the $L^{1}$ approach; that is, by changing the class of uncertainty somewhat, a tremendous gain in computability is suddenly possible. With regard to further progress in robustness theory; we seem to now be fighting against the NP-hardness barrier. That is, many of the open problems for linear systems involve nonlinear dependence on uncertain parameters and are known to be NP-hard. However, nothing rules out the possibility that a slight reformulation of the robustness problem will no longer be NP-hard while still capturing the essence of the control problem of interest.

\section{Major challenges}

I will address this issue in a narrow way by only discussing one aspect. In the U.S., economic conditions are such that hiring in the control field has reached a virtual standstill. If this trend does not reverse, we will soon see very few graduate students opting to study control; in fact, many colleagues argue that such a trend is already underway. Without the injection of new blood into the field, we are in grave danger. On the other hand, there are colleagues who would argue that much of this phenomenon is attributable to the fact that the control field is now mature. Hence, some would argue that reduced entry into the field may be the appropriate equilibrium. My personal opinion, however, is that many of the major problems in the field are yet to be solved - especially problems involving nonlinearity. 
Bensoussan A. (INRIA, Rocquencourt, France)

Past research

Stochastic control with partial information, non linear filtering, controllability, $H_{\infty}$ theory.

Major open problems

Real time control of large sytems.

Major challenges

Reducing the gap between theory and practice.

Keeping a minimum common core.

Incorporating computer science methods, without loosing leadership. 
Byrnes C. I. (Systems Sciences and Mathematics, Washington University, St Louis, USA)

Past research

1. Feedback stabilization of continuous-time, nonlinear finite dimensional systems.

2. Nonlinear optimal control with convex and with nonconvex performance measures.

3. Tracking, regulation and disturbance attenuation for nonlinear systems.

4. Feedback control of discrete-time, nonlinear finite dimensional systems.

5. Feedback control of linear and nonlinear distributed parameter systems.

6. Nonlinear dynamics of filtering algorithms.

7. The stochastic partial realization problem.

Major open problems

Developing a systematic methodology for taking advantage of genuinely nonlinear effects to shape, or influence, the response of complex dynamical systems.

\section{Major challenges}

1. To respond to the growing pressure by society on universities to transfer ideas into products.

2. To incorporate the remarkable advances in materials sciences and engineering into new models and paradigms for control systems.

3. The merger between computers and communications has ushered in what is usually called the information age. No other technological development has caught this level of popular attention and enthusiasm. We must become leader, in both education and research, in the multimedia communications revolution rather than followers.

4. To incorporate biological systems into our world view at a similar level as we have incorporated electrical and mechanical systems. 
Caines P. E. (Department of Electrical Engineering and Centre for Intelligent Machines, McGill University, and Canadian Institute for Advanced Research, Montreal, Canada)

\section{Past research}

I have been continuing my work on stochastic adaptive control for time varying and randomly evolving systems, and I have initiated a research program in logic control. The latter involves the formulation and solution of systems and control problems within extended versions of the predicate calculus of logic.

\section{Major open problems}

1. In the area of adaptation I think that:

(a) The original motivating problem of the adaptive control of time varying (possibly stochastic) systems has only been solved in a set of isolated -albeit interesting and valuableclasses of problems. There is as yet no overall theory of the behaviour of adaptive systems for wide classes of time varying systems.

(b) There is not as yet an adequate robust adaptive control theory; this may be due to the fact that there is a complete mismatch between the current mathematical formulations of robust and adaptive control theory.

2. Contemporary systems and control does not have theories that deal with the interaction of continuous systems (often the controlled system) and discrete systems (often the controller). So systems and control faces the problems of hybrid systems (in the broadest interpretation of this term) as a set of Major open problems. In my view their formulation and solution will require a fusion of systems and control, logic, and computer science techniques.

\section{Major challenges}

The control engineering of systems with large discrete components (either in the systems themselves or in the regulators, or both) will be a principal challenge to control engineering. This will not be just the classical issue of discrete and continuous time and continuous and quantized variables, but will involve a profound analysis of the interaction of dynamical and computational systems. In my view, this will make the problems of the relations between (1) syntax (grammar) and axiomatics and (2) semantics (meaning and in particular, in the present case, dynamical realization) central conceptual issues in systems and control and its engineering applications. 
Chen H. F. (Institute of Systems Science, Chinese Academy of Sciences, Beijing, China) •

Past research

1. The stochastic adaptive control is designed so that the unknown coefficients, orders and time-delays are consistently estimated and the output tracking errors are minimized for the case where the input-output data are produced by an ARMAX system.

2. The estimation errors for time-varying parameters are shown to be the same order of parameter variations and the noise variance.

3. The convergence and optimality of the extended-least-squares based adaptive trackers are proved and the consistency of parameter estimate is also achieved by using the diminishing excitation technique.

4. Both continuous- and discrete-time systems with unknown coefficients are adaptively stabilized under the condition that the possibly existing common factor of $A(z)$ and $B(z)$ is stable, i.e. under the stabilizability condition.

5. For both continuous- and dicrete-time stochastic systems the adaptive LQG problems are solved without requiring stability of $A$.

6. Stability analysis is given for manufacturing systems with unreliable manufacturing systems. Convergence is proved for perturbation analysis based optimization algorithms for Discrete Event Dynamical Systems (DEDS).

7. Robustness analysis is given for stochastic approximation. Asymptotic efficiency is proved for both continuous- and discrete-time averaged stochastic approximation algorithms with slowly varying gains.

8. Stochastic approximation is used for estimating parameters in nonlinear systems and for proving convergence of recursive algorithms arising from various areas.

Major open problems

1. How to realize robust identification aiming at robust control?

2. Adaptive control for partially observed systems.

3. Identification and adaptive control for the "error in variables" case.

4. Recursive algorithm minimizing performance criteria in $H_{\infty}$ norm.

5. Analysis for least-squares-based adaptive pole assignment.

6. Mathematical set-up for describing general DEDS.

7. To find effective algorithms which almost surely lead to the global minimum of an unknown function observed with noise.

8. Practically applicable methods for controlling nonlinear systems. Recently, Prof. J. Q. Han at the Institute of Systems Science developed a nonlinear PID using the differential signal obtained by a tracking technique rather than direct differentiation. This method seems to be promising in dealing with nonlinear systems. 


\section{Major challenges}

System control scientists have been developing elegant theories for analysing and controlling systems in various descriptions. On the other hand, practical problems arising from engineering, as a rule, do not meet the conditions required in theory, and a sophisticated theory is not easy to be accepted by practitioners. This gap faces the control engineering community. 
Davis M. H. A. (Department of Electrical Engineering, Imperial College of Science and Technology, London, U.K.)

\section{Past research}

1. Theory of piecewise-deterministic Markov processes. Most of stochastic control theory in continuous time concerns the Ito stochastic differential equation model, and this excludes a large body of applied problems in, for example, queueing systems, inventory control, planning and scheduling models and the like, where the basic source of randomness is a sequence of point events rather than continuously-acting "noise". The piecewisedeterministic Markov process model covers all such applications. In my book Markov Models and Optimization I develop a complete stochastic calculus for piecewise-deterministic Markov processes and, based on this, a theory of optimization covering continuous control, optimal stopping and impulse control.

2. A deterministic approach to stochastic optimization. The most characteristic feature of stochastic optimization is the non-anticipativity constraint that decisions must depend only on information available at the time they are taken. It has long been recognized in the stochastic programming world that this is a linear equality constraint that can be enforced by introduction of a suitable Lagrange multiplier. I and collaborators have extended this approach to optimal stopping and stochastic control; effectively, this "reduces" stochastic control to deterministic control and concentrates the stochastic aspects on the search for the right multiplier.

3. Financial models with transaction costs. These lead to fascinating problems of singular stochastic control, some of which we have been able to solve.

\section{Major open problems}

Surely, the "grand challenge" is to make adaptive control work. The subject has been studied for years but in spite of impressive achievements there are still gaping holes in what we can do. There are exact asymptotic theories that are completely non-robust, theories of robust control that are non-adaptive, and various other pieces of the jigsaw puzzle, but no grand synthesis: one needs control strategies for nonlinear systems that guarantee performance by identifying a system model that is "good enough" to keep errors within adequate bounds for robust control.

\section{Major challenges}

Let me dispense the following pieces of perhaps provocatively phrased gratuitous advice to the community. Of course, I don't claim in all cases to be following this advice myself.

1. Get out of the ghetto. Control is not like Physics. It is like Statistics: a service industry that exists to provide solutions to problems in a wide range of application areas. Yet if one peruses the control journals -particularly the more prestigious ones- one encounters far too many papers addressing "internal" questions that, it is obvious, will never be of even indirect interest or relevance to the world outside. This is unhealthy.

2. Go for a wider sphere of applications. Applications of control techniques have been spreading from control of "plants" (process systems, aircraft, etc.) to "plant-wide management" encompassing a whole range of operations research-type issues. Yet apart from the rather isolated Discrete Event Dynamical Systems subculture little of this has been formalized and almost none of it appears in textbooks. A shift in perspective is called for.

3. Make more effective use of computational power. I get the impression -perhaps wronglythat the control community has been rather unambitious in its utilization of modern 
computational resources, compared to, say, physicists. Harnessing this immense potential is certainly another major challenge. 
Davison E. J. (Department of Electrical and Computer Engineering, University of Toronto, Toronto, Canada)

Past research

1. Robust control: attempting to solve the "real stability radius" problem.

2. Adaptive control: attempting to reduce the number of a priori assumptions required for a plant to be controlled.

3. Nonlinear control: attempting to enlarge the class of nonlinear plants to which a solution to the servomechanism problem can be obtained.

4. Large scale system theory: attempting to develop a complete theory of decentralized control.

5. Large flexible space structures: attempting to develop a theory re the control of noncollocated large fexible space structures.

6. Robotic systems: attempting to develop a theory re the control of multi-cooperative robotic systems.

\section{Major open problems}

I believe the field is just in its infancy. In this sense, there are many significant open problems in control, e.g.

1. Generalization of Kharitonov theory to the matrix case.

2. Development of a tractable algorithm to determine existence conditions re stabilization of $(C, A, B)$ using output feedback.

3. Nonlinear system theory - "almost everything".

\section{Major challenges}

1. Existing theory often lags control system practice. There is a desperate need to develop new appropriatre relevant theory!

2. To demonstrate and verify new theory using hardware and application studies.

3. To relate control system theory and engineering to other fields in a more effective way, e.g. computer sciences, biological systems, management science, signal analysis and processing, banking systems, "intelligent" system theory. 
Fliess M. (Ecole Nationale Supérieure d'Electricité, CNRS, Gif sur Yvette, France)

Past research

I have been working on an algebraic theory of linear and nonlinear systems. Besides many theoretical results, this setting has caused new insights on more classic domains, such as sliding modes (joint work with H. Sira-Ramirez). Let me also mention dynamic feedback linearization (joint work with J. Levine, P. Martin and P. Rouchon), which led to flat systems. They provide the solution of several practical problems, like motion planning.

\section{Major open problems}

Control theory today is divided in several quite independent branches. Is it possible to find a kind of unification which permits a deeper understanding? I hope that the above mentioned algebraic theory, which is strongly related to Willems' behavioral approach, could be of some help.

\section{Major challenges}

Some of the existing theories do not seem to be really helpful in applications. A major challenge is to understand why and to propose some remedies. 
Goodwin G. C. (Department of Electrical and Computer Engineering, University of Neweastle, Newcastle, Australia)

\section{Past research}

Over the past 5 years I have had the priviledge of working (in collaboration with students and colleagues) on the following problems:

1. Adaptive Control: Continuous time stochastic adaptive control; Hysteresis switching adaptive control of MIMO systems; Constrained adaptive control via differential games.

2. Computational Issues in Control and Estimation: The Delta Operator; High speed digital signal processing and control.

3. Control System Design Issues: Partial decoupling of MIMO systems; Integral constraints for MIMO systems.

4. Estimation: Continuous time lattice algorithms; Parameter estimation with missing data; Estimation of periodic ARMA models.

5. Fault Detection: Use of approximate models; Application to gas turbine aircraft engines.

6. Filter Design: Integral constraints on linear filter design; Fundamental design trade-offs in filtering, prediction and smoothing.

7. Identification for Control: Stochastic embedding approach to undermodelling; Bias distribution in least squares estimation.

8. Nonlinear Systems: Internal model control with saturating actuators; Input disturbances in feedback linearization; Complementarity constraints for sensitivity in nonlinear systems.

9. Optimization: Connecting discrete and continuous $H_{\infty}$; Loop transfer recovery using biased controllers.

10. Sampled Data Systems: Frequency domain sensitivity functions; Robustness of generalized sampled data holds and periodic controllers; Duality of hybrid optimal regulator and hybrid optimal filter; Reconstruction of band limited signals from periodic non-uniform samples.

11. Applications: Ship motion prediction; Aircraft manoeuvre autopilots; Thermal camber control in rolling mills; Bloom caster secondary cooling; Molecular beam epitaxy; Fatigue testing of aircraft empennages; Nonlinear identification and control of mould level in continuous bloom casting; Zinc coating mass estimation and control; Automobile engine control.

12. Products: UNAC - A control system design and implementation platform providing easy access to an integrated suite of tools for real-time data measurement, model building, controller synthesis, simulation, on-line prototyping, bumpless transfer and final plant implementation.

\section{Major open problems}

My impression is that there is much more we can learn about design as applied to practical control problems. Of particular importance would be a better understanding of the fundamental performance limitations that arise from such issues as start-up and product change; smooth and non-smooth nonlinearities; non-minimum phase behaviour; disturbances and noise; MIMO interactions; approximate models; amplitude and slew rate limited actuators; and constrained 
sensor technology.

\section{Major challenges}

The question the control community needs to ask is whether or not industry is "beating down our doors" to get access to our ideas. It is certainly true that strong theory has been, and must remain, the cornerstone of Control Engineering. However, our greatest challenge is to better - communicate the relevance of this theory to practical design issues. 
Kimura H. (Department of Mechanical Engineering, Osaka University, Osaka, Japan)

Past research

My main research interest in the last five years has been in establishing a unified theoretical framework of $H_{\infty}$ control which connects the classical interpolation approach to modern state-space theory. I have been working with a systematic method of solving the $H_{\infty}$ control problem based on chain-scattering representation and J-lossless factorization which turns out to be closely related to classical circuit theory. Another area I have been interested in is the interpolation approach to identification for robust control. I have also been involved in a number of applications of robust control to real systems.

Major open problems

There are a number of open problems. To list a few:

1. Pole-assignment by output feedback without recourse to heavy mathematics like algebraic geometry.

2. To obtain the minimal order of a stabilizing compensator for a given plant.

3. To derive a simple adaptive control scheme that works for most plants in practice.

4. To identify the largest model set containing all the plants that are consistent with a given data and a priori information.

5. To derive an efficient numerical method for solving nonlinear $H_{\infty}$ control problem.

\section{Major challenges}

1. It is important to establish the intrinsic necessity of adaptive controllers based on the clear demonstration of the performance limit of linear time-invariant controllers.

2. An interesting challenge is to formulate the control and estimation problem for quantum mechanical systems. For instance, it is extremely interesting to recover the phase of wave functions utilizing the additional information during the measurement process.

3. It may be possible to establish, as an extension of robust control, a new design philosophy in which the quality of our knowledge is explicitly taken into account. 
Kučera V. (Institute of Information Theory and Automation, Czech Academy of Sciences, Praha, Czech Republic)

Past research

1. Descriptor linear systems: eigenstructure assignment, feedback realization of feedforward compensators, and $\mathrm{H}_{2}$ optimal control.

2. Factorization approach to control system synthesis: parametrization of admissible controllers, selection of best or robust controllers by solving Diophantine equations.

3. Parallels between state-space and transfer-function techniques in linear control system design, with emphasis on the LQG and $H_{2}$ designs.

\section{Major open problems}

The limits of static output feedback in altering the dynamics of linear systems.

The study of output feedback has a long history spanning decades. There have been many partial results on pole placement; unfortunately, these are of generic sort and rely on conditions that do not always hold. There are few conditions for stabilizability that make intuitive sense, and no easy-to-use noniterative algorithms that give stabilizing output feedback gains. And what the static output feedback can do with respect to altering the eigenstructure of linear systems is an open problem. A result like Rosenbrock's fundamental theorem of state feedback is the ultimate goal.

\section{Major challenges}

Classical design of control systems was based on fitting frequency responses. Modern control theory has brought in mathematical models and formulated optimal control problems. However there is one difficulty: the design is valid for the nominal model only. Future control theories will have to handle families of models to account for mismodelling and produce reliable control systems. 
Laub A. J. (Department of Electrical and Computer Engineering, University of California, Santa-Barbara, USA)

Past research

Creating new algorithms and improving old ones (e.g., with respect to efficiency or numerical reliability) for key numerical problems arising in control engineering (such as Riccati equations, Lyapunov equations, frequency response); trying to get better estimates of problem condition and solution accuracy for a variety of key control problems.

\section{Major open problems}

Creating algorithms and software for various key control-theoretic problems that estimate how accurate computed solutions are.

\section{Major challenges}

Developing the next generation of (parallel and vector) algorithms, computer codes, and user interfaces for computer-aided control system design. 
Ljung L. (Department of Electrical Engineering, Linköping University, Linköping, Sweden)

Past research

1. To better understand detection, tracking, and adaptation mechanisms in on-line estimation algorithms.

2. To understand how to come to grips with evaluating the total error when estimating models of real plants.

3. Various preliminary attempts to handle the problem of dealing with the discrete (hybrid, logic, sequential) elements in control systems.

Major open problems

To come up with a model concept which incorporates the discrete (logical, hybrid, etc.) elements in our traditional model world; a concept that should turn out to have design and synthesis power.

Major challenges

To have a broad and open mind about control and feedback: Not to focus on the traditional control loops and leave the rest to the computer scientists and software engineers. 
McFarlane A. J. G. (Heriot-Watt University, Edinburgh, U.K.)

Past research

I have effectively become a full-time administrator with a deep interest in the general and social implications of technology, and so the honest answer is "none". I did however attempt a synoptic overview of the field and its future in the essay "Information, Knowledge and Control" published in "Essays on Control: Perspectives in the Theory and its Applications", edited by H. L. Trentelman and J. C. Willems, Birkhäuser, 1993.

\section{Major open problems}

I quote from the above reference: "The biggest single challenge facing Control, or indeed any other modern technology, is how to:

1. Organise and make interactively accessible the large and growing amount of objective formal knowledge which is available.

2. Develop a coherent, communicable, synoptic overview of the subject which can be used to illuminate and organise this knowledge.

3. Make the knowledge useable by practitioners - to provide them with a powerful toolkit.

4. Relate the knowledge to reality, that is to experimental and practical investigations."

\section{Major challenges}

1. To develop a coherence among the wide range of disparate approaches in current use.

2. To relate more closely to cognate fields - information sciences, computational and cognate sciences, artificial intelligence, and physiology and neurophysiology.

3. To absorb the implications of parallel processing for applied control. 
Picci G. (Department of Electrical and Computer Engineering, University of Padova, Padova, Italy)

Past research

Mostly on stochastic systems theory, modeling of uncertain systems and signals (stochastic realization theory) and applications of the theory to estimation and identification in a statistical . framework.

\section{Major open problems}

I believe that identification (i.e., automatic model building of real systems from observed data) is a very important area which is still in its infancy and does need a lot of research. Most of the basic issues are in my opinion still open or poorly understood like, for example,

1. The meaning and limitations of statistical methods in dealing with data encountered in engineering applications. What are viable alternatives to statistical thinking. What classes of models are most appropriate and what are the fundamental limitations of identification, e.g. how "precise" can an estimate be? which features (parameters and/or auxiliary variables) of a model does it make sense to "estimate" from the data?

2. The question of (stochastic) model approximation, i.e. choosing model complexity viewed in relation to the use/purpose of the model, say control forecasting etc.

3. The question of uncertainty bounds and of robustness of the estimated model with respect to fluctuations in the data and to unmodelled variables (disturbances).

4. So far the main paradigm in identification has been to reduce the problem to linear regres. sion (perhaps recursive linear regression), which is a rather elementary idea indeed. Many popular books on the subject are essentially about how to phrase identification as a linear regression problem or are structured around this idea. This approach has been criticized recently. More general classes of models (say Errors-in-Variables or Factor-Analysis-models) should be considered.

\section{Major challenges}

There is a naturally built-in imperative in the field: to go towards more pervasive and sophisticated automatization. This means moving more and more towards autonomous systems, i.e. systems capable of completing complicated tasks with only a minimum of very high level commands from a human operator. For example autonomous mobile robots, autonomous navigation and transportation systems etc. All these systems must be able to construct, update and interpret an on-line model of the environment in which they operate on the basis of measurements provided by suitable sensors. New sensors (like computer vision) and ultrafast computers make building of these systems a real possibility. In this context I believe that identification, estimation and modelling theory will have to play an important role.

This may seem like trespassing onto the realm of "Artificial Intelligence". However I believe that the basic faith behind "Artificial Intelligence" that heuristics and phenomenological imitation of animal behavior would cheaply yield solutions to difficult technological problems is fading out. The visible successes of artificial intelligence (besides creating an impressive vocabulary and very catching buzzwords) seem still to be very very few. On the other hand, the central ideas for a rational approach to autonomous systems are based on feedback, identification and adaptation, and do certainly belong to the control and systems engineering culture. A solid theory for the design of systems capable of on-line testing, modeling and identifying (i.e. "learning" about) the environment will be a primary need in this area. 
Poznyak A. S. (Department of Electrical Engineering, CINVESTAV, Mexico, on leave from the Institute of Control Sciences of the Russian Academy of Sciences, Moscow)

Past research

1. Learning automata theory. Analysis of behaviour of probability recurrent reinforcement schemes in random environments with application to the multimodal function optimization.

2. Stochastic convex optimization and recurrent identification of $A R M A X$ processes under dependent noises. Convergent and asymptotic normality analysis of recurrent procedures with nonlinear transformations of residuals: minimax Huber's approach.

3. Analysis of identification algorithms of nonstationary linear processes. Analysis of ARMAX models with nonstationary parameters.

4. Adaptive control of Markov chains.

5. Robust control under mixed (external and internal) uncertainties. Robust control for linear systems with time-varing parameters under deterministic and stochastic nature of noises.

6. Artificial neural networks. Optimization of a number of nodes in networks with small inner disturbances.

Major open problems

1. To give a mathematically correct solution of the adaptive filtering problem.

2. To construct information inequalities (like Cramer-Rao inequalities in statistics) for adaptive control problems to stop useless competition in invention of new "bad" (which do not reach these bounds) adaptive algorithms.

3. Design of robust-adaptive and adaptive-robust control theories for finite and infinite dimensional systems.

4. Development of game theory with many participants under internal a priori uncertainties.

Major challenges

The control engineering community will be faced with the necessity

1. to reorganize the system of education (to suggest more intensive programs) in the field of control theory as each year the distance between the student - programs level and the level of leading science schools is increasing;

2. to participate in the joint research in investigation of thermo-nuclear-controllable processes because of a coming world energetic crisis;

3. to participate in the joint research in medical investigation of human health problems to help medicine to apply a systems approach to these investigations which can consider the human body as the unique controllable mechanism. 
Skelton R. E. (School of Aeronautical and Astronautical Engineering, Space Systems Control Laboratory, Purdue University, West Lafayette, USA)

Past research

Beginning in the early 1980 's, students in the School have helped develop a complete theory for covariance control to determine the set of all state covariance matrices (or quadratic Lyapunov functions). This theory reduces to the linear algebra problem

$$
\begin{aligned}
B G C+(B G C)^{T}+Q & =0 \\
B^{\perp} Q B^{\perp T} & =0 \\
C^{T \perp} Q C^{T \perp T} & =0 \\
f(B, C, Q) & =0
\end{aligned}
$$

and the solution $G(B, C, Q, Z)$ is explicitly given in terms of free parameters $\mathrm{Z}$. The same results (1-3) apply to the case where the equality in (1) is replaced by an inequality, simply by replacing the equalities in (2), (3) by inequalities, and deleting condition (4). This single linear algebra problem solves 18 different control problems: (1) All stabilizing controllers, (2) All covariance (upperbound) controllers, (3) State space mu upperbound controllers, (4) All $H_{\infty}$ controllers, (5) $\mathrm{H}_{2}$ guaranteed cost controllers, (6) All $L_{\infty}$ controllers (that guarantee $L_{\infty}$ bounds in the presence of $L_{2}$ disturbances), (7) Robust $L_{\infty}$ controllers, (8) Robust $H_{\infty}$ controllers, (9) Robust $H_{2}$ controllers. These nine problems have continuous and discrete time counterparts, adding to a total of 18 problems that all reduce to a single linear algebra problem (see new book to appear, A Unified Algebraic Approach to Control Design, by Skelton, Iwasaki, and Grigoriadis).

\section{Major open problems}

These are open problems:

1. For a linear system to be driven by noisy actuators, what is the optimal number of actuators to achieve minimum variance in the output signal?

2. What is the minimal complexity (by some measure such as memory) controller required to guarantee a specified level of output performance?

3. Can optimal control problems be implemented as data-based controllers rather than modelbased?

4. A fundamental limitation of robust control theory (which often leads to a high gain) is the presumption that the model errors are independent of the input (and indeed that a transfer function exists for the real systems, independent of the qualities of the input). Available control techniques do not come to grips with the fact that the modeling problem and the control problem are not independent problems, and must necessarily be treated as iterative procedures. Can a scientific iterative approach be developed for modeling and control?

\section{Major challenges}

I believe we are near the limits of performance that can be obtained by the isolated theories and the sequential steps of plant design, model development, sensor/actuator selection, control design, controller implementation (signal processing). The control community must develop scientific procedures for integrating (and yes iterating) these disciplines in a scientific way. Such a system design theory would probably not be some linear combination of existing theories, because some of the premises upon which the isolated theories are developed (modeling and control for example) collapse when integrated with the control problem. 
Tsypkin Ya. Z. (Institute of Control Science, Russian Academy of Sciences, Moscow, Russia).

Past research

Lately I have been working at the problem of dynamic plant control under bounded uncertainty. The main idea is to take into consideration a priori information about uncertainty caused by the change of parameters and characteristics of a plant and by external disturbances in the control law in order to eliminate their influence on the control processes. This can be obtained by introducing internal models of a certain nominal plant and some vague internal models of deviations from a nominal plant. The theory of robust stability, which was founded by the theorem of $V$. L. Kharitonov plays the main- role in the realization of such robust nominal systems.

\section{Major open problems}

Among the main open problems of modern control theory are synthesis problems of robust, or in other words weakly sensitive to parameter changes and external diturbances, systems with variable parameters and nonlinear systems. The solution of these problems is closely connected with the development of the theory of robust stability and synthesis automatisation on the basis of analytic and computer methods.

\section{Major challenges}

I hope that there will appear new points of growth in control theory, such as those that took place earlier: in the 50's - optimal control of R. Kalman, A. S. Pontryagin, R. Bellman; in the 60 's - frequency domain criteria of nonlinear systems absolute stability of V. M. Popov; in the $70-80$ 's - robust stability criteria of V. L. Kharitonov and their generalizations, and also various variants of robust control. It is difficult to forecast what new trends will appear in the 90's, but they are to appear. Then the control engineering community will become younger and will enthusiastically provide further development of control theory. 
Varaiya P. P. (Department of Electrical Engineering and Computer Science, University of California, Berkeley, USA)

Past research

Problems of hybrid control: development of theory for verification of properties of hybrid system, and applications to operating systems for real-time control and to automated highways.

Major open problems

In my opinion, one urgent issue is how to formalize symbol-processing, embedded, controllers within a continuous process.

Major challenges

How to use some of the key concepts of control -information, feedback, algorithms- in the formulation of a control architecture for realistic, complex systems. 
Vidyasagar M. (Centre for Artificial Intelligence and Robotics, Bangalore, India)

Past research

During the past five years, I have been looking at neural networks from the standpoint of dynamical system theory. I have been trying to understand whether the various claims made on behalf of neural networks have any mathematical validity. Specifically, I wished to understand whether neural networks are indeed useful for performing discrete optimization, and whether neural networks can indeed "generalize" from given data. My investigations into discrete optimization led me to prove a general theorem on the minimum-seeking properties of analog neural networks with discontinuous dynamics. In short, this theorem states that it is possible to construct a differential equation (which has an obvious interpretation as a neural network) with the following property: Solution trajectories of the equation starting from "almost all" initial conditions will converge, within a finite amount of time, to a local optimum of the objective function. Now I am trying to evolve a meaningful theory of computational complexity for problem solving using analog differential systems. Earlier work focused for the most part on memoryless analog systems. My research on the ability of neural networks to generalize led me to work on learning theory, as epitomized by the PAC (probably approximately correct) framework. In this framework, I managed to prove a couple of interesting results on when a class of concepts is learnable under an arbitrary family of probability distributions. I have also been encouraged to turn my lecture notes on PAC learning theory into a monograph, which I am now trying to do.

\section{Major open problems}

I hesitate to answer this question, because $I$ have never been a mainstream control theorist at any time during my career. With that caveat, let me say that an area that $I$ find quite exciting is that of building intelligent control systems. This is a much abused term, because most so-called intelligent control systems are in fact stupider than a well-designed conventional feedback control system. Nevertheless, questions such as "What does learning on the basis of past experience mean?", "How does a system generalize on the basis of experiment?", "What is the intrinsic difficulty of a given task?" and so on, are good candidates for a formal treatment using the methodologies (though not the specific results) of control theory. Another interesting question is: "How can one combine differential/difference equations with logical switches so as to enhance performance?" In some sense, this is the central question of intelligent control.

\section{Major challenges}

In my view, the major challenge faced by the control engineering community at present is coping with the interface between control and computing. Several persons have attempted to approach this interaction through the methodology of discrete-event systems, but in my view, most of the existing approaches do not really address the "real" issues. For instance, it is a nontrivial task merely to ensure that the control law perfected by the control engineer has actually been implemented correctly. I believe we should begin to understand at least the basic ideas involved in the use of CASE tools, for example, and what "guaranteed" correctness of programs really means. Another challenge faced by us is the need to understand how the evolution of semiconductor hardware (e.g., Field Programmable Gate Arrays, cellular neural networks) will affect the way in which control laws are designed. Up to now, the design of control laws has proceeded on the assumption that a linear time-invariant control law is easier to implement than any other. What happens if this fundamental assumption is challenged? 
Willems J. C. (Mathematics Institute, University of Groningen, Groningen, The Netherlands)

Past research

During the past 5 years, I have been working on the development of the behavioral approach to systems and control. In this framework, a dynamical system is simply viewed as a family of trajectories, called the behavior. It can be described in various ways, usually by means of behavioral equations. Signal flow graphs, transfer functions, state space models, and the like, are a far distance removed from typical models obtained in practice. Models obtained from first principles, by tearing and zooming, will usually contain many algebraic relations involving latent variables, in addition to the manifest variables which the model aims at describing. These are the principles on which the behavioral approach is built.

Specific questions studied often center on representation issues, identification by approximation, model reduction, etc. Recently we have used this framework in order to study control problems. The classical signal flow graph input/output setting is often cumbersome and unnatural. The alternative approach which we have developed takes interconnection of systems as the basic idea for control and dynamic response improvement. This picture incorporates subsystem design, passive control, and active control.

\section{Major open problems}

I suspect that the question about the major open problem in control and systems theory is meant in the way mathematicians think of open problems, as Fermat's last theorem, the Riemann hypothesis, or the invariant subspace conjecture. If the question is meant in this sense, then it is both an inappropriate and an irrelevant one. Usually such open questions are readily answered, often after reformulation, or quickly abandoned. The art in control theory is to shape new questions, to introduce new concepts, to build new paradigms.

Among the areas in which there is a need for new paradigms, I would name the modelling of uncertainty, abandoning the unrealistic stochastic approach, and less simplistic than small gains or hard parameter bounds. A second area is to treat dynamic modelling - not just identification - as an integral part of control theory, thus creating an area of research which develops flexible foundations at the same time as it pushes into new frontiers. Part of this is the development of a nonlinear and infinite dimensional system theory which does not start from an input/output or a first order differential equation setting. A final area is the theory of integrated design in which the design of the controller and the plant are part of the same picture.

\section{Major challenges}

I believe that the major challenge for the control engineering community lies in taking advantage of advanced model based control concepts in order to improve performance of process control systems. While good control can often be achieved by very simple controllers, and as a means of improving performance, is overshadowed by advances in instrumentation, I believe that there are many instances where redesign of classical controllers using modern mathematical concepts is worthwhile. The availability of inexpensive hardware and software in order to implement such methods makes this a practical proposition. This challenge includes pinpointing those applications which can be effectively described by means of mathematics, a process which all too often requires making simplifications which makes subsequent refined analysis and optimization not a very meaningful activity. 
Wonham W. M. (Department of Electrical Engineering, University of Toronto, Canada) .

Past research

Qualitative (logico-linguistic) problems in the modelling and control of discrete-event systems. Specifically:

1. Timed transition models based on a temporal logic framework (with J.S. Ostroff, T.J. Ho, M. Lawford).

2. Dișcrete-event systems architecture in the Ramadge/Wonham framework, including hierarchical supervision (with H. Zhong, K.C. Wong) and decentralized control and coordination with partial observations (with F. Lin, K. Rudie, P. Kozak).

3. Vector discrete-event systems (with Y. Li, N.Q. Huang, S.L. Chen).

4. Timed Ramadge/Wonham models (with B.A. Brandin, F. Lin).

5. Discrete-event systems with infinite string behavior (with J.G. Thistle).

Major open problems

Most problems involving large systems are open. The most significant practical problem area may well be real-time computer control, with the concomitant problems of software specification and correctness, and system reliability. From the theoretical viewpoint the fundamentals of modelling and control of large, many-sorted structures are still very unclear - for instance, the concepts of state and state-transition, and the architectural principles of system decomposition, . modularization and aggregation.

\section{Major challenges}

To gain a much better understanding of system complexity - what is and what is not tractable and what can and cannot be done. Clearly the control science and engineering community will have to move closer to computer science and engineering, but without (I hope) losing faith in rigorous problem formulation or giving up formal synthesis. Perhaps the community should be somewhat more adventurous and outward-looking than it has tended to be in the past, more willing to absorb and exploit ideas from other subcultures. For instance, the current interest in "intelligent controls" (however diffuse all that may seem at the moment) is a definite sign of vitality. 


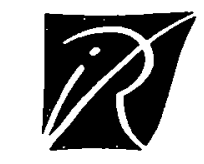

Unité de recherche INRIA Rocquencourt

Domaine de Voluceau - Rocquencourt - B.P. 105 - 78153 Le Chesnay Cedex (France)

Unité de recherche INRIA Lorraine - Technopôle de Nancy-Brabois-Canpus scientifique 615. rue du Jardin Botanique - B.P. $101-54602$ Villers les Nancy Cedex (France)

Unite de recherche INRIA Remes - IRISA. Campus universitaire de Beaulieu 35042 Rennes (Cedex (France)

Unité de recherche I: ¿RIA Rhône-Alpes 46, avenue Félix Viallet - 380.3। Grenoble Cedex I (France)

Unité de recherche INRIA Sophia Antipolis - 2004, route des Lucioles - B.P. 9.3 - 96902 Sophia Autipolis Cedex (France)

Éditcur

INRIA - Domaine de Voluceau - Rocquencourt - B.P. $105-781.53$ Le Chesnay Cedex (France)

ISSN $0249-6399$ 